\title{
Primary malignant lymphoma of the uvula
}

Takeshi Kusunoki ${ }^{1 *}$, Hirotomo Homma ${ }^{1}$, Yoshinobu Kidokoro ${ }^{1}$, Aya Yanai ${ }^{1}$, Ryo Wada ${ }^{2}$ and Katsuhisa Ikeda ${ }^{3}$

${ }^{1}$ Department of Otorhinolaryngology, Juntendo University of Medicine, Shizuoka Hospital, Japan

${ }^{2}$ Departement of Pathology, Juntendo University of Medicine, Shizuoka hospital, Japan

${ }^{3}$ Department of Otorhinolaryngology, Juntendo University of Medicine, Faculty of Medicine, Japan

\begin{abstract}
We experienced a very rare case of primary malignant lymphoma of the uvula. The patient was an 80-year-old Japanese woman with a one month history of oral discomfort. She had a uvular mass occupying the oropharyngeal space and received surgical treatment. Follicular lymphoma was diagnosed by immunohistochemical staining. In the general examination, PET-CT showed no abnormal accumulation in the body, and the marrow chromosome examination results were normal. From the above results, our case was considered stage IA (UICC) and we added postoperative radiotherapy (33.6Gy). At 3 years after the radiation therapy, no recurrence or metastasis was found.
\end{abstract}

\section{Introduction}

Malignant neoplasms of the uvula are rare, and the most common histopathology is squamous cell carcinoma [1]. Moreover, malignant lymphoma of the uvula is exceedingly rare. We encountered a primary malignant lymphoma of the uvula and describe its clinical findings, MRI and treatment.

\section{Case report}

A 80-year-old Japanese woman presented with a one month history of oral discomfort. Physical examination revealed uvular swelling with a smooth surface that was elastic hard. This uvular mass occupied the oropharyngeal space (Figures 1 and 2). In the laryngopharynx, Waldeyer's tonsillar ring and neck, tumors and swelling of the neck lymph nodes could not be found by visual palpation, MRI, CT or echo. The biopsy examination of the uvula swelling revealed only inflammation of the mucous membranes. The patient received surgical treatment and the uvular tumor was extirpated by electric knife and coagulation with a safety margin of $2 \mathrm{~mm}$ (Figure 3 ). There was little intraoperative bleeding. Postoperatively, there was no wound infection, snuffling voice or dysphagia.

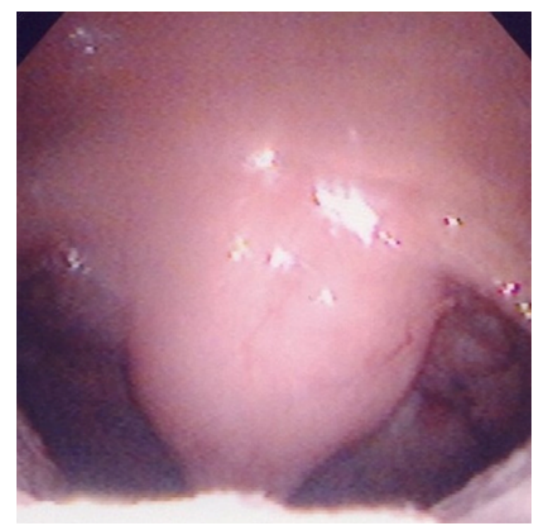

Figure 1. A uvular mass showed a smooth surface that was elastic hard.
Histological examination showed atypical lymphocytes by $\mathrm{H} \& \mathrm{E}$ staining and suspected malignant lymphoma (Figure 4). On the edge surrounding a mass of malignant cells could be found. For this reason, we consulted pathologists and a hematologist; follicular lymphoma was diagnosed by immunohistochemical staining (CD10:+, CD20:+, bcl-2:+) . In the general examination, PET-CT revealed no abnormal accumulation in the body, and the marrow chromosome examination was normal. From the above results, our case was considered stage IA (UICC) and we added postoperative radiotherapy (33.6Gy). At 3 years after the radiation therapy, no recurrence or metastasis was found.

\section{Discussion}

Extra-nodular malignancies with initial symptoms in the oral cavity are rare. Their occurrence is $2.2 \%$ of malignant tumors and $3.5 \%$ of malignant tumors of the oral cavity [2]. Sugiyama [3] reviewed 125 extra-nodular malignancies with initial symptoms in the oral

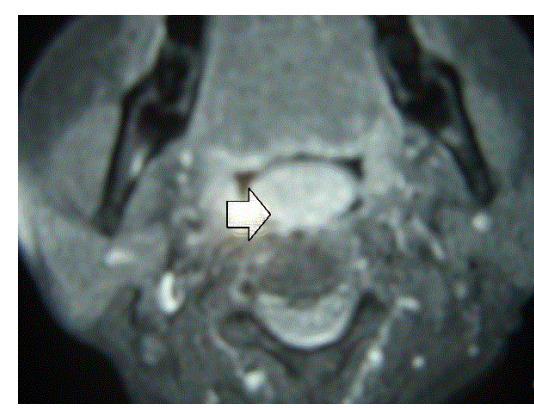

Figure 2. MRI demonstrated a mass (an arrow) occupying the oropharyngeal space.

Correspondence to: Dr. Takeshi Kusunoki, Department of Otorhinolaryngology, Juntendo University of Medicine, Shizuoka Hospital, 1129 Nagaoka Izunokunishi, Shizuoka 410-2295, Japan; Fax: +81-55-948-5088; E-mail: ttkusunoki001@aol.com

Key words: malignant lymphoma, uvula, immunohistochemical staining

Received: January 05, 2017; Accepted: January 25, 2017; Published: January 28, 2017 


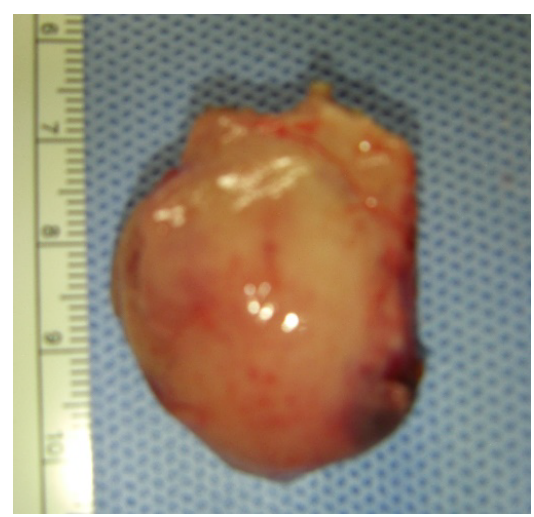

Figure 3. A specimen with a safety margin of $2 \mathrm{~mm}$ did not reveal malignant cells in the surrounding edges of thetumor.

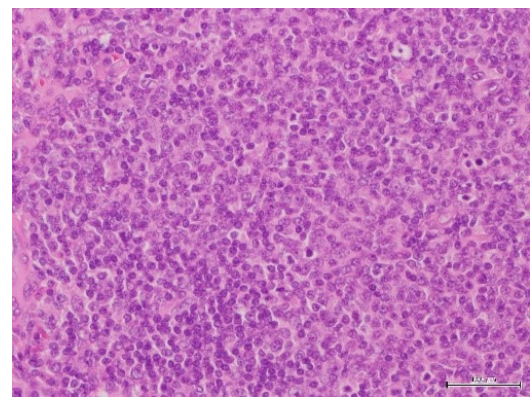

Figure 4. Histopathological findings of the uvular tumor showed proliferating atypical lymphocytes with small oval cells (H\&E staining).

cavity reported in Japan. The primary site with the highest incidence was the gingiva in $47.2 \%$ of oral malignancies. In histopathology, $73 \%$ of the cases were diffuse large B cell lymphoma and $12 \%$ were MALT lymphoma Our case was follicular lymphoma, which has a low incidence of $2.4 \%$. Malignant lymphoma is classified by various histopathologic types and is often difficult to diagnose. In our case, Pre-operatively, the biopsy examination of the uvula tumor revealed only inflammation without malignancy. But, follicular lymphoma could be diagnosed by immunohistochemical staining (CD10:+, CD20:+, bcl-2:+) postoperatively . We suggest that an exact diagnosis by the malignant lymphoma classification would require resect enough surgical specimens for immunological study by combining key antibodies. Moreover, for the treatment as well as diagnosis it would be necessary to consult pathologists and hematologists. Mac Manus and Hoppe [4] recommended that the treatment of choice for early stage(I and II) follicular lymphomas as our case would be radiation therapy (35-50Gy). The results of their radiation therapy demonstrated actuarial survival rates of $5,10,15$ and 20 years were $82 \%, 64 \%$ and $35 \%$,respectively. Our case stage IA (UICC) underwent postoperative radiotherapy (33.6Gy). At 3year after the radiation therapy, no recurrence or metastasis was found.

Primary malignant lymphoma of the uvula is very rare. However, a few reports in the world have presented malignant lymphoma of the uvula accompanied by lymphoma of the neck, breast or stomach $[1,5,6]$. Therefore, we followed up and checked for recurrence and metastasis in the whole body and oropharyngeal area by PET-CT and hematological findings.

\section{Declarations}

\section{Author's contributions}

Takeshi Kusunoki MD: diagnosis and therapy, composition of this case report

\author{
Hirotomo Honma MD: therapy \\ Yoshinobu Kidokoro MD: therapy \\ Aya Yanai MD: therapy \\ Ryo Wada MD: Pathological diagnosis
}

Katsuhisa Ikeda MD: composition of this case report

\section{Consent for publication}

Consent for publication on the clinical details, images and histopathological examination was obtained for this case report. A copy of this document is available for review by the Editor of this journal.

\section{Acknowledgements}

The authors thank Mr. Brent Bell for checking English of the manuscript.

\section{References}

1. Walker R, Heffelfinger R (2012) Low-grade B-cell lymphoma presenting as a uvular mass. Ear Nose Throat J 91: E22-24.[Crossref]

2. Sugiyama S, Iwai T, Oguri S, Nakajima H, Koizumi T, et al. (2016) Clinical study of extra-nodal malignant lymphoma with initial symptoms in oral cavity. Japanese Journal of Head and Neck Cancer 42: 339-344.

3. Eisenbud L, Sciubba J, Mir R, Sachs SA (1983) Oral presentations in non-Hodgkin's lymphoma: a review of thirty-one cases. Part I. Data analysis. Oral Surg Oral Med Oral Pathol 56: 151-156.[Crossref]

4. Mac Manus MP, Hoppe RT (1996)Is radiotherapy curative for stage I and II low grade follicular lymphoma? Results of a long term follow-up study of patients treated at Atandford University. J Clin Oncol 14: 1282-1290.

5. Singh A, Singh RK (2014) Right molar approach for uvulectomy of secondary nonhodgkins lymphoma of uvula. Indian J Anaesth 58: 358-359.[Crossref]

6. Okabe S, Kuriyama Y, Kawanishi Y, Yahata N, Miyazawa K, et al. (2001) MALT lymphoma originating in breast and uvula. Leuk Lymphoma 41: 461-463.[Crossref]

Copyright: (C2017 Kusunoki T. This is an open-access article distributed under the terms of the Creative Commons Attribution License, which permits unrestricted use, distribution, and reproduction in any medium, provided the original author and source are credited. 